\title{
Random Evolutions with Underlying Semi-Markov Processes
}

\author{
By
}

\author{
Robert P. KERTZ*
}

\section{Introduction}

In [9] Griego-Hersh introduced random evolutions with underlying finite-state Markov chains and formulated a representation of the solution of an abstract Cauchy problem in terms of the expectation of the random evolution. Extensions of the initial definition and its uses have been completed by various authors and are reported in the surveys of Hersh [13] and Pinsky [27]. In this paper we extend the definition of random evolution to the case in which the underlying process is a semi-Markov process. We prove new representation theorems for solutions of various abstract integral equations in terms of this generalized random evolution. In particular, we use a special type of random evolution with underlying semi-Markov process to give a new representation for the solution of abstract Cauchy problems of the type treated by Griego-Hersh and, motivated through this representation, we generate new limit theorems of 'generalized-central-limit-theorem type' for the abstract Cauchy solutions.

In Section one we present background results on Markov renewal processes and semi-Markov processes which we need in this development. In Section two we define the random evolution with underlying semi-Markov process and related notions; we then prove conditioning results for the random evolutions and representation theorems for solutions of abstract integral equations in terms of

Communicated by K. Itô, August 1, 1976.

* School of Mathematics, Georgia Institute of Technology, Atlanta, Georgia 30332, U.S. A. 
expectations of these random evolutions. We end this section with a limit theorem which demonstrates how the operator structure of the random evolution can be combined with the renewal structure of the semi-Markov process, and then give an application of this theorem in storage theory. In Section three we specialize the underlying semi-Markov process to obtain the new representations of the solutions of abstract Cauchy equations; we compare our representation to those in Pinsky [27] and Heath [13], [11]. Using transform analysis and operator theory, we prove new perturbation theorems for the Cauchy solutions with limit as a higher order diffusion. In another paper [21] we extend Mürman's semi-Markov model for Brownian motion [25] by using the concepts in this paper to define a family of random evolutions over the underlying semiMarkov process.

\section{Section 1}

We introduce terminology used in the following sections. The definitions and notations used here are similar to those in [5], [6], [2], and [29]. Let $N=\{0,1,2, \ldots\}, R_{+}=[0, \infty)$, and $\mathscr{R}_{+}=$Borel sets on $R_{+}$. A semi-Markovian kernel on measure space $(E, \mathscr{E})$ is any transition probability from $(E, \mathscr{E})$ into $\left(E \times R_{+}, \mathscr{E} \times \mathscr{R}_{+}\right)$. On probability space $(\Omega, \mathscr{F}, P),\left\{X_{n}\right\}_{n \in N}$ and $\left\{T_{n}\right\}_{n \in N}$ denote stochastic processes, taking values in $E$ and $R_{+}$respectively, and $\left\{\mathscr{F}_{n}\right\}_{n \in N}$ denote sub- $\sigma$-algebras increasing to $\mathscr{F} . \quad(X, T)=\left(\Omega, \mathscr{F}, \mathscr{F}_{n},\left(X_{n}, T_{n}\right), P\right)$ is a Markov renewal process (MRP) over $(E, \mathscr{E})$ with semi-Markovian kernel $Q$ provided $\left(X_{n}, T_{n}\right) \in \mathscr{F}_{n} / \mathscr{E} \times \mathscr{R}_{+}$for each $n \in N$ and

$$
P\left(X_{n+1} \in A, T_{n+1}-T_{n} \in B \mid \mathscr{F}_{n}\right)=Q\left(X_{n}, A \times B\right) \quad \text { a. e. }
$$

for each $n \in N, A \in \mathscr{E}$, and $B \in \mathscr{R}_{+}$. From (1.1) we have that $\left\{T_{n}\right\}$ are increasing; we assume that $T_{0} \equiv 0$, and

$$
\sup _{n \in N} T_{n}=\infty .
$$

For sufficient conditions giving (1.2), see [4] and p. 326, [6]. The (minimal) semi-Markov process $Y=\left\{Y_{t}\right\}_{t \geq 0}$ associated with $\operatorname{MRP}(X, T)$ 
is defined by

$$
Y(t, \omega)=X_{n}(\omega) \text { for } T_{n}(\omega) \leq t<T_{n+1}(\omega) .
$$

For each $x, y \in E$ and $u \in R_{+}$we let $N_{x, y}(u)=\sum_{n ; 0<T_{n} \leq u} I_{\left\{x_{n-1}=x, X_{n}=y\right\}}=$ number of transitions of $X$ from $x$ to $y$ during $(0, u]$, and $N(u)=$ $\sum_{x, y \in E} N_{x, y}(u)$. Note that we are counting transitions from a state to itself in the $X$ process. For $t \in R_{+}$we let $U(t)=t-T_{N(t)}=$ time elapsed since the last 'regenerative epoch'. For $y \in E, u \in R_{+}, \gamma_{y}(u)=$ Lebesgue measure of $\{0 \leq s \leq u ; Y(s)=y\}=$ 'occupation time' of process $Y$ in state $y$ during $(0, u)$. The notation $P_{x}(\cdot)$ represents the conditional probability $P\left(\cdot \mid X_{0}=x\right)$ and $E_{x}(\cdot)=E\left(\cdot \mid X_{0}=x\right)$.

For $n=1,2, \ldots$, let $\theta_{n}: \Omega \rightarrow \Omega$ denote shift operators satisfying $X_{m} \circ \theta_{n}=X_{m+n}, \quad T_{m} \circ \theta_{n}=T_{m+n}-T_{n}$, and $Y_{t+T_{n}}=Y_{t} \circ \theta_{n}$. For $s \in R_{+}$, let $\theta_{s}: \Omega \rightarrow \Omega$ denote shift operators for which on $\left\{T_{k} \leq s<T_{k+1}\right\}, T_{n} \circ \theta_{s}=$ $T_{n+k}-s, Y_{T_{n}} \circ \theta_{s}=X_{n} \circ \theta_{s}=X_{n} \circ \theta_{k}=X_{n+k}=Y_{T_{n+k}}$, and $N(t) \circ \theta_{s}=N(t+s)-$ $N(s)=N(t+s)-k$. Such shift operators will exist, although enlarging the space $\Omega$ may be necessary [29], [2]. The following theorem and corollary follow directly from (1.1) and (1.2) and standard Monotone Class Theorem arguments as in [2].

Theorem 1. 1. Given $\operatorname{MRP}(X, T)=\left(\Omega, \mathscr{F}, \mathscr{F}_{n},\left(X_{n}, T_{n}\right), \theta_{n}, P\right)$ with state space $(E, \mathscr{E})$ and associated $\mathrm{SMP} Y=\left\{Y_{t} ; t \geq 0\right\}$.

(1) The semi-Markov property is equivalent to each of the following conditions, holding a. e. :

$$
P_{x}\left(\left(X_{m}, T_{m}\right) \circ \theta_{n} \in C \mid \mathscr{F}_{n}\right)=P_{\mathrm{x}_{n}}\left(\left(X_{m}, T_{m}\right) \in C\right)=Q\left(X_{n}, C\right)
$$

for each $x \in E, m \geq n \geq 1$, and $C \in \mathscr{E} \times \mathscr{R}_{+}$;

$$
E_{x}\left[H \mid \mathscr{F}_{n}\right](\omega)=E_{\mathrm{X}_{n}(\omega)}[G(\omega, \bullet)]
$$

for $H(\omega)=G\left(\omega, \theta_{n} \omega\right)$ and $G: \Omega \times \Omega \rightarrow R$ bounded, $\mathscr{F}_{n} \times \mathscr{F}$-measurable, $x \in E, n \geq 1$.

(2) The following are equivalent:

$$
P\left(Y_{T_{n}+s} \in A \mid \mathscr{F}_{n}\right)=P_{X_{n}}\left(Y_{s} \in A\right)
$$

for each $s \in R_{+}, \quad n \geq 1, A \in \mathscr{E}$ 


$$
E\left[W \circ \theta_{n} \mid \mathscr{F}_{n}\right]=E_{x_{n}}[W]
$$

for $n \geq 1$ and $W$ any real-valued, bounded, $\sigma\left(Y_{s}, s \in R_{+}\right)$-measurable function.

Here $\sigma(\cdot)$ denotes the $\sigma$-algebra generated by $(\cdot)$. Analogues of (1.6) and (1.7) are also equivalent if we replace $\left\{Y_{t}, t \in R_{+}\right\}$, by the $\left\{U_{\imath}, t \in R_{+}\right\}$process.

Corollary 1. 1. Given MRP $(X, T)$ with SMP $Y$. Then for $x \in E, t \in R_{+}$, on the set $\left\{T_{n} \leq t\right\}$ we have a. e. for $A \in \mathscr{E}, m \geq n$,

$$
\begin{gathered}
P_{x}\left[Y_{t} \in A \mid \mathscr{F}_{n}\right](\omega)=P_{X_{n}(\omega)}\left[Y_{t-T_{n}(\omega)} \in A\right] \\
P_{x}\left[N(t)=m \mid \mathscr{F}_{n}\right](\omega)=P_{X_{n}(\omega)}\left[N\left(t-T_{n}(\omega)\right)=m-n\right] .
\end{gathered}
$$

We will need the following $\sigma$-algebras associated with processes $(X, T)$ and $Y$. For $n \in N, \mathscr{M}_{n}=\sigma\left(X_{0}, \ldots, X_{n}\right) ; \mathscr{G}_{n}=\sigma\left(X_{0}, T_{0}, \ldots\right.$, $\left.X_{n}, T_{n}\right) \subset \mathscr{F}_{n} ;$ and $\mathscr{H}_{s}=\sigma(Y(t), U(t) ; 0 \leq t \leq s)=\sigma\left(N(s), T_{1}, \ldots, T_{N(s)}\right.$, $\left.X_{0}, \ldots, X_{N(s)}\right)$. We set $\mathscr{M}_{\infty}=\sigma\left(\mathscr{M}_{n}, n \in N\right), \mathscr{G}_{\infty}=\sigma\left(\mathscr{G}_{n}, n \in N\right)$, and $\mathscr{H}_{\infty}=\sigma\left(\mathscr{H}_{s}, s \in R_{+}\right)$. We remark that Theorem 1.1 and Corollary 1.1 hold for $\mathscr{F}_{n}$ and $\mathscr{F}$ replaced by $\mathscr{G}_{n}$ and $\mathscr{G}_{\infty}$ respectively. Since the regenerative epochs $\left\{T_{n}\right\}$ are stopping times with respect to $\left\{\mathscr{H}_{s}, s \in R_{+}\right\}$, we set $\mathscr{H}_{T_{n}}=\left\{\Lambda \in \mathscr{F} ; \Lambda \cap\left\{T_{n} \leq t\right\} \in \mathscr{H}_{t}\right.$ for each $\left.t \in R_{+}\right\}$(see [2], p. 32). From the characterization of $\mathscr{H}_{T_{n}}$ given in p. 35 of [2] and p. 86 of [16], one easily sees that $\mathscr{G}_{n}=\mathscr{H}_{T_{n}}$. Finally, we set $\mathscr{J}_{s}=\sigma\left(N(s), T_{1}, \ldots, T_{N(s)}, X_{0}, \ldots, X_{N(s)+1}\right)$.

We note that $X=\left(\Omega, \mathscr{M}_{\infty}, \mathscr{M}_{n}, X_{n}, P\right)$ is a Markov process with state space $(E, \mathscr{E})$ and transition probability $\hat{Q}(x, A)=Q\left(x, A \times R_{+}\right)$, for $x \in E, A \in \mathscr{E}[5]$.

\section{Section 2}

Let $L$ denote a Banach space with norm $\|\cdot\|_{L}$ and topological Borel field $\mathscr{L}$. We let $\left\{\Gamma(x, y, u) ; x, y \in E, u \in R_{+}\right\}$denote bounded, linear operators on $L$ which satisfy $\Gamma(x, y, 0)=I, \Gamma(x, y, u)$ is strongly continuous in $u$, and $\Gamma(x, y, u) f$ is strongly measurable 
in $(x, y)$ with respect to $\mathscr{E} \times \mathscr{E} / \mathscr{L}$. Thus $\Gamma(x, y, u) f$ is $\mathscr{E} \times \mathscr{E} \times \mathscr{R}_{+} / \mathscr{L}$ strongly measurable. We let $\{\Pi(x, y) ; x, y \in E\}$ denote bounded, linear operators on $L$ for which $\Pi(x, y) f$ is $\mathscr{E} \times \mathscr{E} / \mathscr{L}$ strongly measurable.

We define the random evolution (R. E.) with underlying MRP $(X, T)$ to be the random operators $M=\left\{M(t, \omega) ; t \in R_{+}, \omega \in \Omega\right\}$ on $L$ given by

$$
\begin{aligned}
& M(t) f= \Gamma\left(X_{0}, X_{1}, T_{1}\right) \Pi\left(X_{0}, X_{1}\right) \Gamma\left(X_{1}, X_{2}, T_{2}-T_{1}\right) \ldots \\
& \Gamma\left(X_{n}, X_{n+1}, t-T_{n}\right) f \quad \text { for } T_{n}<t<T_{n+1}, n \in N \\
&= \Gamma\left(X_{0}, X_{1}, T_{1}\right) \Pi\left(X_{0}, X_{1}\right) \ldots \\
& \Gamma\left(X_{n-1}, X_{n}, T_{n}-T_{n-1}\right) \Pi\left(X_{n-1}, X_{n}\right) f \\
& \quad \text { for } t=T_{n}, n \geq 1 .
\end{aligned}
$$

Assumption (1.3) guarantees that $N(t)<\infty$; thus the product is well-defined. For each $f \in L$ we have $\left\{M(t) f ; t \in R_{+}\right\}$is $\mathscr{J}_{t} / \mathscr{L}_{-}$ strongly measurable and is strongly continuous at $t \neq T_{n}, n \geq 1$. This definition includes as special cases those definitions of 'random evolutions' in the literature with special underlying MRP given as a continuous-parameter, finite-state Markov chain [9], [19], conservative regular jump process [27], and discrete-parameter Markov chain [18].

Let $\widetilde{L}$ denote the Banach space $\widetilde{L}=\oplus L$ and let $\tilde{L}_{\infty}$ be the subspace of $\tilde{L}$ given by $\widetilde{L}_{\infty}=\left\{\tilde{f}=\left(f_{x}\right)_{x \in E} \in \tilde{L} ; x \rightarrow f_{x}\right.$ is $\mathscr{E} / \mathscr{L}$ measurable and $\left.\sup _{x \in E}|| f_{x} \|<\infty\right\} . \quad \widetilde{L}_{\infty}$ is a Banach space of equivalence classes with norm $\|\tilde{f}\|=\sup _{x \in E}\left\|f_{x}\right\|_{L}$. We define the expectation operators of the random evolution $M$ with underlying $\operatorname{MRP}(X, T)$ as the family of operators $\left\{\widetilde{T}(t), t \in R_{+}\right\}$on $\widetilde{L}_{\infty}$ given by

$$
[\widetilde{T}(t) \tilde{f}]_{x}=E_{x}\left[M(t) f_{Y(t)}\right]
$$

for $\tilde{f} \in \widetilde{L}_{\infty}, x \in E$. Here $Y=\left\{Y(t), t \in R_{+}\right\}$is the SMP associated with $(X, T)$. The expectation here is in the sense of Bochner integrability [14], [1], [9], [28]. The expectation operators are thus well defined if and only if we have for each $t \in R_{+}$,

$$
E_{x}\left[\left\|M(t) f_{Y(t)}\right\|\right]<\infty
$$




$$
M(t, \cdot) f_{Y(t, \cdot)} \text { is strongly measurable. }
$$

See Theorem 3.7.4 of [14]. A sufficient condition for (2.3) to hold is

(2.5) $\{\Gamma(x, y, u)\}_{x, y \in E, u \in R_{+}}$and $\{\Pi(x, y)\}_{x, y \in E}$ are contractions;

another sufficient condition is the combination of (2.6) and (2.7):

$$
\|\Gamma(x, y, u)\| \leq K e^{\lambda u} \text { and } \mid i \Pi(x, y) \| \leq K
$$

for each $x, y \in E, u \in R_{+}$and for some constants $K, \lambda$ independent of $x, y, u$; and

$$
P_{x}\{N(t)=n\} \leq(\beta(t))^{n} \gamma(t) / n !
$$

for each $x \in E, n \in N$ and for finite functions $\beta(t), \gamma(t)$ independent of $x, n$. Condition (2.7) holds, in particular, for MRP's which are either finite-state Markov chains [9] or certain renewal processes [7]. The strong continuity and measurability assumptions on $M$ give (2.4) [9]. In what follows we assume that either (2.5) or (2.6) - (2.7) hold, and hence (2.3) - (2.4) hold. For special operators $\Gamma$ and MRP's $(X, T),\left\{\widetilde{T}(t), t \in R^{+}\right\}$form a semigroup and hence have been called the 'expectation semigroup' of the R. E. $M$ [9].

We define the discrete-parameter expectation semigroup of the R. E. $M$ with underlying $\operatorname{MRP}(X, T)$ as the operators $\left\{\widetilde{T}_{n} ; n \in N\right\}$ on $\tilde{L}_{\infty}$ given by

$$
\left(\widetilde{T}_{n} \tilde{f}\right)_{x}=E_{n}\left[M\left(T_{n}\right) f_{Y\left(T_{n}\right)}\right]
$$

for $\tilde{f} \in \tilde{L}_{\infty}, x \in E$. A uniform-boundedness condition such as (2.6) guarantees existence and boundedness of the operators $\widetilde{T}_{n}$; strong measurability of $\widetilde{T}_{n} \tilde{f}$ with respect to $\mathscr{G}_{n} / \mathscr{L}$ follows as before. We observe that for special types of MRP's such as discrete-parameter MRP's, the discrete-parameter expectation semigroup will be a subset of the expectation operators of $M$. The semigroup appellation will be justified in Theorem 2.2.

We give two structure theorems and a limit theorem: Theorem 2. 1 gives a conditioning result for R. E. $M$; Theorem 2. 2 uses this result to obtain renewal-type equations satisfied by the expectation 
operators and discrete-parameter expectation semigroup associated with R. E. $M$; Theorem 2.3 gives a limit theorem for a special type of R. E. associated with R. E. $M$.

Theorem 2. 1. For the random evolution $M=\left\{M(t), t \in R_{+}\right\}$with underlying $\operatorname{MRP}(X, T)$ we have a. e.

$$
E_{x}\left[M\left(T_{n}+s\right) f_{Y\left(T_{n}+s\right)} \mid \mathscr{G}_{m}\right]=M\left(T_{m}\right) E_{X_{m}}\left[M\left(T_{n-m}+s\right) f_{\left.Y_{\left(T_{n-m}\right.}+s\right)}\right] ;
$$

and on $\left\{T_{n} \leq t\right\}$ we have a. e.

$$
E_{x}\left[M(t) f_{Y(t)} \mid \mathscr{G}_{n}\right](\omega)=M\left(T_{n}\right)(\omega) E_{X_{n}(\omega)}\left[M\left(t-T_{n}(\omega)\right) f_{Y\left(t-T_{n}(\omega)\right)}\right]
$$

Proof. We give the proof of (2.10); the proof of (2.9) is analogous. For each $x \in E, t \in R_{+}, n \in N$, we have on $\left\{T_{n} \leq t\right\}$ that a. e.

$$
\begin{aligned}
& E_{x}\left[M(t) f_{Y(t)} \mid \mathscr{G}_{n}\right](\omega)=\sum_{m=n}^{\infty} E_{x}\left[M(t) f_{Y(t)} \cdot I_{\left\{T_{m} \leq t<T_{m+1}\right\}} \mid \mathscr{G}_{n}\right](\omega) \\
& =\sum_{m=n}^{\infty} M\left(T_{n}\right)(\omega) E_{x}\left[\Gamma\left(X_{0}, X_{1}, T_{1}\right) \Pi\left(X_{0}, X_{1}\right) \ldots\right. \\
& \ldots \Gamma\left(X_{m-n}, X_{m-(n-1)}, t-T_{n}(\omega)-T_{m-n}\right) f_{Y\left(t-T_{n}(\omega)\right)} \circ \theta_{n} \omega \\
& \left.\quad \cdot I_{\left(T_{m-n} \leq t-T_{n}(\omega)<T_{m-(n-1)\}}\right.} \circ \theta_{n} \omega \mid G_{n}\right](\omega) \\
& =\sum_{m=n}^{\infty} M\left(T_{n}\right)(\omega) E_{X_{n}(\omega)}\left[M\left(t-T_{n}(\omega)\right) f_{Y\left(t-T_{n}(\omega)\right)} .\right. \\
& \left.\cdot I_{\left\{T_{m-n} \leq t-T_{n}(\omega)<T_{m-(n-1)}\right]}\right] \\
& =M\left(T_{n}\right)(\omega) E_{X_{n}(\omega)}\left[M\left(t-T_{n}(\omega)\right) f_{\left.Y_{\left(t-T_{n}(\omega)\right)}\right] .}\right.
\end{aligned}
$$

Here we have used that the operators $\{\Gamma(x, y, u)\}$ and $\{\Pi(x, y)\}$ are bounded and linear, and hence are distributive with conditional expectation $[28]$; that $(X, T)$ satisfies $(1.5)$; and that $\Gamma(x, y, 0)=\mathrm{I}$ for each $x, y \in E$.

Remark. From Theorem 2.1 we essentially have also that the process $\left\{Z(t), t \in R_{+}\right\}$given by

$$
Z(t)=\Gamma\left(X_{n}, X_{n+1}, t-T_{n}\right) f_{Y(t)}
$$

for $T_{n} \leq t<T_{n+1}$, is a Markov renewal process with auxiliary paths (MRPAP), where for this concept it has been required that $E$ is countably additive [29], or equivalently, a semiregenerative process 
[6] or cumulative process [30].

Theorem 2. 2. Given the R. E. $M=\left\{M(t), t \in R_{+}\right\}$with underlying $\operatorname{MRP}(X, T)$ having state space $(E, \mathscr{E})$ and semi-Markovian kernel $Q$. Then we have

(1) the $\tilde{L}_{\infty}$-valued function $u(\cdot, t)=\widetilde{T}(t) \tilde{f}, t \in R_{+}$, satisfies

$$
\begin{aligned}
u(x, t) & =\int_{E} Q(x, d y \times(t, \infty)) \Gamma(x, y, t) f_{x} \\
& +\int_{E \times[0, t]} Q(x, d y \times d s) \Gamma(x, y, s) \Pi(x, y) u(y, t-s)
\end{aligned}
$$

for $\tilde{f} \in \widetilde{L}_{\infty}, x \in E$;

(2) the family of operators $\left\{\widetilde{T}_{n}, n \in N\right\}$ is a discrete-parameter semigroup for which the $\widetilde{L}_{\infty}$-valued function $w(\cdot, n)=\widetilde{T}_{n} \tilde{f}, n=1,2, \ldots$, satisfies

$$
w(x, n)=\int_{E \times R_{+}} Q(x, d y \times d t) \Gamma(x, y, t) \Pi(x, y) w(y, n-1)
$$

for each $x \in E, n \geq 1$; and

(3) the $\widetilde{L}_{\infty}$-valued function $v(\cdot ; n, s)=\widetilde{T}_{n} \tilde{T}(s) \tilde{f}$ satisfies

(2. 14) $v(x, n, s)=\int_{E \times R_{+}} Q(x, d y \times d r) \Gamma(x, y, r) \Pi(x, y) v(y ; n-1, s)$ for each $x \quad E, s \in R_{+}$, and $n=1,2, \ldots$

Proof. (1) For $x \in E, t \in R_{+}, \tilde{f} \in \tilde{L}_{\infty}$, we have

$$
\begin{aligned}
u(x, t) & =(\tilde{T}(t) \tilde{f})_{x}=E_{x}\left[M(t) f_{Y(t)}\right] \\
& =E_{x}\left[M(t) f_{Y(t)} ; T_{1}>t\right]+E_{x}\left[M(t) f_{Y(t)} ; T_{1} \leq t\right] .
\end{aligned}
$$

First,

$$
\begin{aligned}
E_{x}\left[M(t) f_{Y(t)} ; T_{1}>t\right] & =E_{x}\left[\Gamma\left(X_{0}, X_{1}, t-T_{0}\right) f_{Y(t)} ; T_{1}>t\right] \\
& =E_{x}\left[\Gamma\left(x, X_{1}, t\right) f_{x} ; T_{1}>t\right] \\
& =\int_{E} Q(x, d y \times(t, \infty)) \Gamma(x, y, t) f_{x} .
\end{aligned}
$$

Second, we obtain from (2.10) that

$$
\begin{aligned}
E_{x}\left[M(t) f_{Y(t)} ; T_{1} \leq t\right] & =E_{x}\left[M\left(T_{1}\right) \cdot(\omega) \cdot\right. \\
& \left.E_{x_{1}(\omega)}\left[M\left(t-T_{1}(\omega)\right) f_{Y\left(t-T_{1}(\omega)\right)}\right] ; T_{1}(\omega) \leq t\right]
\end{aligned}
$$




$$
\begin{aligned}
& =\int_{E \times[0, t]} Q(x, d y \times d s) \Gamma(x, y, s) \Pi(x, y) E_{y}\left[M(t-s) f_{\mathrm{Y}(t-s)}\right] \\
& =\int_{E \times[0, t]} Q(x, d y \times d s) \Gamma(x, y, s) \Pi(x, y) u(y, t-s) .
\end{aligned}
$$

Thus (2. 12) follows.

(2) The operators $\left\{\widetilde{T}_{n}, n \in N\right\}$ form a semigroup since $\left(\widetilde{T}_{0} \tilde{f}\right)_{x}=f_{x}$ from $\Gamma(x, y, 0)=I$, and since we have from (2.9) that for $m, n \in N$

$$
\begin{aligned}
\left(\tilde{T}_{n} \widetilde{T}_{m} \tilde{f}\right)_{x} & =E_{x}\left[M\left(T_{n}\right)\left(\widetilde{T}_{m} \tilde{f}\right)_{x_{n}}\right]=E_{x}\left[M\left(T_{n}\right) E_{X_{n}}\left[M\left(T_{m}\right) f_{X_{m}}\right]\right] \\
& =E_{x}\left[M\left(T_{n}\right) E_{x}\left[M\left(T_{m}\right) f_{X_{m}} \circ \theta_{n} \mid \mathscr{G}_{n}\right]\right] \\
& =\left[E_{x}\left(M\left(T_{n+m}\right) f_{X_{m+n}}\right]=\left(\widetilde{T}_{n+n} \tilde{f}\right)_{x} .\right.
\end{aligned}
$$

Equations (2.13) and (2.14) are proved through application of (2.9) with the $\sigma$-algebras $\mathscr{G}_{1}$ and $\mathscr{G}_{n}$ and $\mathscr{G}_{1}$ respectively.

We give a limit theorem which demonstrates how the operator structure of the random evolution may be combined with the renewaltheoretic property of the Markov renewal process. We let $\rho>0$ be parameters; we use $\rho \downarrow 0$. For each $\rho>0,\left\{Y^{\rho}(t), t \geq 0\right\}$ denotes a semi-Markov process over $(E, \mathscr{E})$ with semi-Markov kernel $Q^{\rho}$ and associated $\operatorname{MRP}\left(X^{\rho}, T^{\rho}\right)$. We let $\tau^{\rho}$ denote an $\mathscr{H}_{t}$-stopping time and introduce iterates $\tau_{0}^{\rho}=0, \tau_{1}^{\rho}=\tau^{\rho}$ and $\tau_{n}^{\rho}=\tau^{\rho} \circ \theta_{\tau_{n-1}^{\rho}}+\tau_{n-1}^{\rho}$. For each $t \geq 0$, we define $N^{\rho}(t)=\sup \left\{n \geq 0 ; \tau_{n}^{\rho} \leq t\right\}$ and assume $N^{\rho}(t)<\infty$ a. e. We let $\left\{M^{\rho}(t), t \geq 0\right\}$ denote a R. E. on Banach space $L$ with underlying $\operatorname{MRP}\left(X^{\rho}, T^{\rho}\right)$, as in (2.1). We define the operator $S_{\rho}$ on $\widetilde{L}_{\infty}$ by

$$
\left(S_{\rho} \tilde{f}\right)_{y}=E_{y}\left[M^{\rho}\left(\tau^{\rho}\right) f_{Y^{\rho}\left(\tau^{\rho}\right)}\right]
$$

for $\tilde{f}=\left(f_{x}\right)_{x \in E} \in \widetilde{L}_{\infty}$. We observe that from the semi-Markov property we have $\left(S_{\rho}^{k} \tilde{f}\right)_{y}=E_{y}\left[M^{\rho}\left(\tau_{k}^{\rho}\right) f_{Y^{\rho}\left(r_{k}^{\rho}\right)}\right]$. When writing $f$ to denote an element of $\tilde{L}_{\infty}$, we mean $\tilde{f}=(f)_{x \in E}$ for $f \in L$.

Theorem 2. 3. We make the following assumptions.

(i) There exists $\omega \geq 0, M \geq 1$ constants for which

$$
|| S_{\rho}^{k}|| \leq M e^{\alpha \rho k} \quad k=1,2, \ldots ;
$$


(ii) For some dense subset $D$ of $L$, we let

$$
\lim _{\rho \downarrow 0} \rho^{-1}\left(S_{\rho}-I\right) f=V f
$$

for every $f \in D$. We assume that

$$
\left(\lambda_{0}-V\right) D \text { is dense in } L
$$

for some $\lambda_{0}$ with $\operatorname{Re} \lambda_{0}>\omega$.

Then there exists a strongly continuous semigroup $U(t)$ on $L$ with infinitesimal operator $\bar{V}$, the closure of $V$, and with $\|U(t)\| \leq M e^{\omega t}$. We have that

$$
\lim _{\rho \downarrow 0} S_{\rho}^{t / \rho} f=U(t) f
$$

where $\rho \downarrow 0$ so that $t / \rho$ takes integer values.

If, in addition, we have

(iii) $S_{\rho}$ is a contraction operator and

$$
E\left[\left(N_{\rho}(t)-t / \rho\right)^{2}\right]=O(t / \rho) \quad \text { as } \rho \downarrow 0
$$

or

(iii)' (2. 20) holds and $E\left[e^{r N^{\rho(t) \rho}}\right] \leq B e^{r t}$, as $\rho \downarrow 0$; then

$$
\lim _{\rho \downarrow 0} E\left[S_{\rho}^{N^{\rho}(t)} f\right]=U(t) f .
$$

Proof. For existence of the semigroup $U(t)$, use (2.18). For (2. 19), apply Theorem 5.3, p. 93 in Pazy [26] or equivalently, Theorem 3.6, p. 511 in Kato [17]. We prove (2.21) under (i), (ii), and (iii)' by using an argument similar to one in Theorem 3. 4. 4 of Pinsky [27]. The proof of (2.21) under (i), (ii), (iii) similar. On $\left\{N^{\rho}(t)<t / \rho\right\}$, we have

$$
\begin{aligned}
& \left\|S_{\rho}^{N^{\rho}(t)} f-S_{\rho}^{t / \rho} f\right\|_{L_{\infty}}=\left\|S_{\rho}^{N^{\rho}(t)}\left(I-S_{\rho}^{t / \rho-N_{\rho}(t)}\right) f\right\| \\
& \leq M e^{\omega \rho N^{\rho}(t)}\left\|\left(I-S_{\rho}^{t / \rho-N^{\rho}(t)}\right) f\right\| \\
& =M e^{\omega \rho N^{\rho}(t)}\left\|\left(I+S_{\rho}+\ldots+S_{\rho}^{t / \rho-N_{\rho}(t)-1}\right)\left(I-S_{\rho}\right) f\right\| \\
& \leq M e^{\omega \rho N^{\rho}(t)} M\left|t / \rho-N_{\rho}(t)\right| e^{\omega \rho\left(t / \rho-N_{\rho}(t)\right)}\left\|\left(I-S_{\rho}\right) f\right\| \\
& =M^{2} e^{\omega t}\left|t / \rho-N_{\rho}(t)\right|\left\|\left(I-S_{\rho}\right) f\right\| .
\end{aligned}
$$

Similarly, we have on $\left\{N^{\rho}(t) \geq t / \rho\right\}$,

$$
\left\|S_{\rho}^{N^{\rho}(t)} f-S_{\rho}^{t / \rho} f\right\|_{L_{\infty}} \leq M^{2} e^{\omega \rho N^{\rho}(t)}\left|N^{\rho}(t)-t / \rho\right|\left\|\left(S_{\rho}-I\right) f\right\| .
$$


Thus

$$
E\left\{\left\|S_{\rho}^{N^{\rho}(t)} f-S_{\rho}^{t / \rho} f\right\|\right\} \leq C e^{\omega t} \rho E\left\{\left(N^{\rho}(t)-t / \rho\right)^{2}\right\}^{1 / 2}
$$

and (2.21) follows from (2.2) and (2.19).

Remark. We prove a similar result under less restrictive operator bounds in Lemma 3.5. This type of convergence result is used as a basis for proving perturbation theorems for solutions of integral equations tending to solutions of diffusion or higher-order-diffusion type equations. See Theorem 2.2, Application 3.1, and [13], [27].

Application 2. 1. We present an application of Theorem (2.3) to storage theory. We are given the following data:

(i) $(X, T)=\left(\Omega, \mathscr{F}, \mathscr{F}_{n},\left(X_{n}, T_{n}\right), P\right)$ is an MRP over $\left(R_{+}, B\left(\mathscr{R}_{+}\right)\right)$ with semi-Markovian kernel $Q$ and associated semi-Markov process $\left\{Y(t) ; t \in R_{+}\right\}$;

(ii) $r: R_{+} \times R_{+} \rightarrow R_{+}$is a jointly continuous function with $r$ and $\frac{\partial r}{\partial z}$ bounded and such that, for each $x \in R_{+}, r(x, 0)=0, r(x, \cdot)$ is non-decreasing, and $\frac{\partial r}{\partial z}(x, \cdot)$ is continuous;

(iii) $k: R_{+} \times R_{+} \rightarrow R_{+}$is a function with $k, \frac{\partial k}{\partial y}$, and $\frac{\partial^{2} k}{\partial y^{2}}$ jointly measurable, with $\frac{\partial k}{\partial y}$ and $\frac{\partial^{2} k}{\partial y^{2}}$ bounded, and such that, for each $x \in R_{+}, k(x, 0)=0$ and $\frac{\partial^{2} k}{\partial y^{2}}(x, \cdot)$ exists and is continuous and

(iv) element $z_{o}$ in $R_{+}$.

Consider the following equation :

$$
Z(t)=z_{o}+\sum_{n \in N ; T_{n+1} \leq t} k\left(X_{n}, X_{n+1}\right)-\int_{0}^{t} r\left(X_{N(s)}, Z(s)\right) d s
$$

for $t \in R_{+}$. This equation is used in storage theory to model the content of the reservoir of a dam; $Z(t)$ is the content of the reservoir at time $t ; z_{o}$ is the initial content of the reservoir; $\sum_{n \in N ; T_{n+1} \leq t} k\left(X_{n}, X_{n+1}\right)$ represents input during time $[0, t]$; and $\int_{0}^{t} r\left(X_{N(s)}, Z(s)\right) d s$ represents output during time $[0, t]$ under release rule $(r(\bullet)$. For a special case of (2.23) see Çinlar ([5], p. 435). 
We let $q(x, z, t)$, for $x, z, t \in R_{+}$, denote the unique solution of the equation

$$
q(x, z, t)=z-\int_{0}^{t} r(x, q(x, z, s)) d s
$$

for $x, z, t \in R_{+}$. We take $L=\hat{C}\left(R_{+}\right)$, the continuous functions on $R_{+}$which vanish at infinity, and define families of contraction operators $\{\Pi(x, \bar{x})\}_{x, \dot{x} \in R_{+}}$and families of strongly continuous semigroups $\left\{\Gamma(x, \bar{x}, \tau) ; \tau \in R_{+}\right\}_{x, \bar{x} \in R_{+}}$on $L$ by

$$
\begin{aligned}
& \Pi(x, \bar{x}) f(z)=f(z+k(x, \bar{x})) \\
& \Gamma(x, \bar{x}, \tau) f(z)=f(q(x, z, \tau))
\end{aligned}
$$

for each $x, \bar{x} \in R_{+}$. Under the conditions imposed on $r$ and $k, \Pi$ and $\Gamma$ satisfy the measurability conditions of Section two. The R. E. $M=\left\{M(t, \omega) ; t \in R_{+}, \omega \in \Omega\right\}$, defined through (2.1) from (2. 25) and $\operatorname{MRP}(X, T)$, satisfies $M(t) f(z)=f(Z(t))$, where $Z(0)=z$.

We wish to apply Theorem 2.3 to determine the behavior of the content process as the magnitudes $X_{n}$ and the time increments $T_{n+1}-T_{n}$ of the MRP $(X, T)$ approach zero. More precisely, for each $\rho>0$, we let $\left(X^{\rho}, T^{\rho}\right)$ be the MRP with semi-Markov kernel given by $Q^{\rho}(x,[0, r] \times[0, t])=Q(x,[0, r / \rho] \times[0, t / \rho])$, so that, for $h$ measurable, $\int Q^{\rho}(x, d y \times d s) h(x, y, s)=\int Q(x, d y \times d s) h(x, \rho y, \rho s)$; and, for each $\rho>0$, we define the content process $\left\{Z^{\rho}(t) ; t \in R_{+}\right\}$ as in (2.23) from $r, k, z_{o}$, and $\operatorname{MRP}\left(X^{\rho}, T^{\rho}\right)$. We wish to investigate the behavior of $Z^{\rho}(t)$ as $\rho \downarrow 0$.

We give the background for application of Theorem (2.3) in this setting. For each $\rho>0$ we define R. E. $M^{\rho}=\left\{M^{\rho}(t) ; t \in R_{+}\right\}$as in (2.1) from (2.25) and $\operatorname{MRP}\left(X^{\rho}, T^{\rho}\right)$ and we let $\tau_{n}^{\rho}=T_{n}^{\rho}$, for all $n \in N$. We observe that $M^{\rho}(t) f(z)=f\left(Z^{\rho}(t)\right)$, for $Z^{\rho}(0)=z$, and that $\left(S_{\rho} f\right)_{x}(z)=\int Q(x, d y \times d s) f(q(x, z, \rho s)+k(x, \rho y))$. It is clear that $S_{\rho}$ is a contraction operator and thus satisfies (2.16). We assume in what follows that the following condition is satisfied:

(2.27) given any $\varepsilon>0$, there is a compact set $\Lambda \subset R_{+} \times R_{+}$for

$$
\text { which } \sup _{x \in R_{+}} \int_{\Lambda^{c}} Q(x, d y \times d s)(s+y)<\varepsilon .
$$


We let $D=\left\{f ; f, f^{\prime} \in \hat{C}\left(R_{+}\right)\right\}$and define $(V f)_{x}$ for $f \in D$ by

$$
(V f)_{x}(z)=\left(-r(x, z) b(x)+\frac{\partial k}{\partial y}(x, 0) \mu(x)\right) f^{\prime}(z)
$$

where

$$
\mu(x)=\int Q(x, d y \times d s) y \text { and } b(x)=\int Q(x, d y \times d s) s .
$$

Then under the conditions on $r(\cdot)$ and $k(\cdot)$ together with (2.27), we have that (2.17) and (2.18) hold. We obtain from (2.19) that $\lim _{\rho \downarrow 0} \sup _{x, z}\left|E_{x}\left[f\left(Z^{\rho}\left(T_{t / \rho}^{\rho}\right)\right)\right]-u(x, z, t)\right|=0$ where $Z^{\rho}(0)=z$ and $u(x, z, t)=(U(t) f)_{x}(z)$ satisfies

$$
\frac{\partial u}{\partial t}(x, z, t)=\left(-r(x, z) b(x)+\frac{\partial k}{\partial y}(x, 0) \mu(x)\right) \frac{\partial u}{\partial z}(x, z, t)
$$

$$
u(x, z, 0)=f(z) \text {. }
$$

Hence $Z^{\rho}\left(T_{t / \rho}^{\rho}\right)$ converges weakly as $\rho \downarrow 0$ and $t / \rho \uparrow \infty$ (through the integers) to the solution $w(x, z, t)$ of (2.29) satisfying the initial condition $w(x, z, 0)=z$. If, in addition, $E_{x}\left[\left(N^{\rho}(t)-t / \rho\right)^{2}\right]=O(t / \rho)$ as $\rho \downarrow 0$, we obtain

$$
\lim _{\rho \downarrow 0} \int E_{x}\left[f\left(Z^{\rho}\left(T_{N_{\rho}(t, \omega)}^{\rho}\right)\right)\right] P_{x}(d \omega)=u(x, z, t)
$$

where $u(x, z, t)$ satisfies $(2.29)-(2.30)$.

In the analysis of the solution of (2.29) - (2.30) for fixed $x \in R_{+}$, three cases arise: $v(x)>0, v(x)=0$, and $v(x)<0$ where $v(x)=$ $\inf _{z}\left\{-r(x, z) b(x)+\frac{\partial k}{\partial y}(x, 0) \mu(x)\right\}$. In each case, $\xi(x, \cdot)=-r(x, \cdot) b(x)$ $+\frac{\partial k}{\partial y}(x, 0) \mu(x)$, is a non-increasing function with $\frac{\partial k}{\partial y}(x, 0) \mu(x)$ $=\xi(x, 0) \geq \xi(x, z) \geq v(x)$; the characteristics of the equation fill the region $R_{+} \times R_{+}$, are defined by the equation $\dot{z}=-\xi(x, z)$, and vary sharply from one case to another. It is of interest to note that in the special case of $Q(x, d y \times d s)=G(d s) \gamma(d y)$ and $k(x, y)=y$ Cinlar [5] shows that the behavior of the content process $Z(t)$, as $t \rightarrow \infty$, separates into the same three cases $(v>0$, $v=0$, or $v<0$ where $\left.v=\inf _{z}\{-r(z) b+\mu\}\right)$. If $v>0, P_{x}\left(\lim _{t \rightarrow \infty} Y(t)=\right.$ $+\infty)=1$; if $v<0, \lim _{t \rightarrow \infty} P_{x}\left[Y\left(T_{N(t)}\right) \in A\right]=\nu(A)$, where $\nu$ is an 
invariant measure for a Markov chain formed from $Z(\cdot)$; and if $v=0$, he conjectures that a $\sigma$-finite, non-finite invariant measure exists, but that $\lim _{t \rightarrow \infty} P_{x}[Y(t) \in A]=0$ for all bounded $A \in \mathscr{B}\left(\mathscr{R}_{+}\right)$.

We note that for the content process with $Q(x, d y \times d s)=\gamma(d y)$ $\lambda e^{-d s} d s$ and $k(x, y)=y$ the analogue of (2.29) was given as Expression (2.18) in ([19], p. 38).

\section{Section 3}

In this section we let $\left\{\left(X_{n}, T_{n}\right), n \in N\right\}$ be a MRP for which $X=\left\{X_{n}, n \in N\right\}$ is a Markov chain with state space $E=\{1,2, \ldots, m\}$ and transition probabilities $\left\{p_{i j}\right\}_{1 \leq i, j \leq m}$ and for which

$$
P\left\{T_{n+1}-T_{n}>t \mid \mathscr{M}_{\infty}\right\}=\exp \left(-\eta\left(X_{n}\right) t\right)
$$

for each $n \in N, t \in R_{+}$and $\eta(j)>0, j \in E$.

Theorem 3.1. Given the $\operatorname{MRP}(X, T)$ as above with associated SMP $Y=\left\{Y_{t} ; t \in R_{+}\right\}$. Then $Y=\left(\Omega, \mathscr{H}_{\infty}, \mathscr{H}_{s}, Y_{s}, P\right)$ is a standard Markov process. In particular, $Y$ satisfies

$$
P_{x}\left[Y_{t} \circ \theta_{s} \in A \mid \mathscr{H}_{s}\right]=P_{Y(s)}\left[Y_{t} \in A\right]
$$

for each $s, t \in R_{+}, A \in \mathscr{E}, x \in E$. The regenerative epochs $\left\{T_{n}, n \in N\right\}$

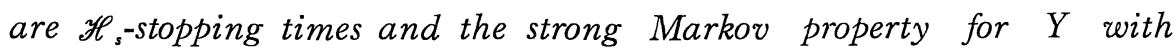
respect to $\sigma$-algebras $\mathscr{H}_{s}, s \in R_{+}$holds at these times.

Proof. With slight modifications, the proof is that for regular step processes as given in [27]. See also [23].

An important point to note here is that the random variables $\left\{T_{n}\right\}$ are stopping times with respect to the $\sigma$-algebras $\left\{\mathscr{H}_{s}\right\}$ but not necessarily with respect to the $\sigma$-algebras $\sigma\left(Y_{r}, 0 \leq r \leq s\right)$. In our setting we are able to distinguish behavior at those epochs at which a 'transition' is made from a state to itself.

Theorem 3.2. Given $\operatorname{MRP}(X, T)$ as above and operators $\left\{\Gamma(x, y, u) ; x, y \in E, u \in R_{+}\right\}$and $\{\Pi(x, y) ; x, y \in E\}$ as in Section two with the additional restrictions that for each $x \in E, u \in R_{+}$, 


$$
\Gamma(x, y, u)=\Gamma(x, u), \text { independent of } y,
$$

and for each $x \in E$,

$$
\Gamma(x, \cdot) \text { is a semigroup. }
$$

\section{Then}

(1) the random evolution $M=\left\{M(t) ; t \in R_{+}\right\}$satisfies

$$
E_{x}\left[M(t) \circ \theta_{s} f_{Y(t)} \circ \theta_{s} \mid \mathscr{H}_{s}\right]=E_{Y(s)}\left[M(t) f_{Y(t)}\right]
$$

for each $s, t \in R_{+}, x \in E$;

(2) the expectation operators $\left\{\widetilde{T}(t) ; t \in R_{+}\right\}$form a strongly continuous semigroup on $\tilde{L}_{\infty}$; and

(3) the $\widetilde{L}_{\infty}$-valued functions $u(\cdot, t)=\widetilde{T}(t) \tilde{f}$ satisfy the initial-value problem (IVP)

$$
\begin{aligned}
\frac{\partial u}{\partial t}(i, t) & =A_{i} u(i, t)-\eta_{i} u(i, t)+\sum_{j=1}^{m} p_{i j} \eta_{i} \Pi_{i j} u(j, t) \\
u(i, 0) & =f_{i}
\end{aligned}
$$

for $1 \leq i \leq m, t \in R_{+}$where $f_{j} \in \mathscr{D}\left(A_{j}, \Pi_{i j} ; i \in E\right)$, the domains of $A_{j}, \Pi_{i j}, 1 \leq i, j \leq n$, and $A_{i}$ generates the semigroup $\Gamma(i, \bullet)$.

Proof. (1) The necessary measurability and the proof for (3.5) follows from our choice of $\sigma$-algebras $\left\{\mathscr{H}_{s} ; s \in R_{+}\right\}$, the form of $M(t)$, the assumption (3.1), and the Markov-type property (3.2) for the $Y$ process.

(2) From the definition of $\left\{\tilde{T}(t), t \in R_{+}\right\}$we have that $\tilde{T}(0)=I$. The semigroup property follows from

$$
\begin{aligned}
(\widetilde{T}(s) \widetilde{T}(t) \tilde{f})_{x} & =E_{x}\left[M(s) E_{x}\left[M(t) \circ \theta_{s} f_{Y(t)} \circ \theta_{s} \mid \mathscr{H}_{s}\right]\right] \\
& =E_{x}\left[M(s+t) f_{Y(t+s)}\right]=(\tilde{T}(t+s) \tilde{f})_{x},
\end{aligned}
$$

where we have used (3.5) the $\mathscr{H}_{s} / \mathscr{L}$ measurability of $M(s) g$, properties of the shift operator $\theta_{s}$, and the semigroup property of $\Gamma(i, \cdot), i \in E$.

(3) We obtain that $u(i, t)=(\widetilde{T}(t) \tilde{f})_{i}=E_{i}\left[M(t) f_{\mathrm{Y}(t)}\right]$ satisfies (3.6) as in [27]. This method of proof uses (2.12) to obtain

$$
\frac{d u}{d t}(i, 0)=A_{i} f_{i}-\eta_{i} f_{i}+\sum_{j=1}^{m} p_{i j} \eta_{i} \Pi_{i j} f_{j}
$$


and that $\left\{\widetilde{T}(t), t \in R_{+}\right\}$is a semigroup, which leaves invariant the domain of its infinitesimal generator.

Corollary 3. 1. Given strongly continuous semigroups $\left\{\Gamma_{i}(u) ; u \in R_{+}\right\}$ with infinitesimal generators $A_{i}, 1 \leq i \leq m$. Suppose $\left\{u_{i}(t), i \in R_{+}\right\}$is the $\widetilde{L}_{\infty}$-valued solution of the initial-value problem

$$
\begin{aligned}
& \frac{\partial u_{i}(\mathrm{t})}{\partial t}=A_{i} u_{i}(t)+\sum_{j=1}^{m} a_{i j} \dot{u}_{j}(t) \\
& u_{i}(0+)=f_{i} \in \mathscr{D}\left(A_{i}\right)
\end{aligned}
$$

for $1 \leq i \leq m, t \in R_{+}$, and real constants $\left\{a_{i j}\right\}_{1 \leq i, j \leq m}$. Then $\left\{u_{i}(t)\right\}$ has representation

$$
u_{i}(t)=E_{i}\left[\Gamma\left(X_{0}, T_{1}\right) \ldots \Gamma\left(X_{N(t)}, t-T_{N(t)}\right) f_{Y(t)} \cdot \Pi_{1 \leq i, j \leq m}\left(\pi_{i j}\right)^{N_{i j}(t)}\right]
$$

where the real parameters $\left\{\pi_{i j}\right\}_{1 \leq i, j \leq m}$ and the parameters $\left\{\eta_{i}\right\},\left\{p_{i j}\right\}$, $1 \leq i, j \leq m$, for the MRP $(X, T)$ with associated SMP $Y$ are chosen so that

$$
\begin{aligned}
a_{i j} & =\eta_{i}\left(p_{i i} \pi_{i i}-1\right) & & \text { for } i=j \\
& =\eta_{i} p_{i j} \pi_{i j} & & \text { for } i \neq j .
\end{aligned}
$$

Remarks. There are two other representations of the solution $\left(u_{i}(t)\right)$ to IVP's of form (3.7). In the setting of (3.7) with $A_{i}=v_{i} \frac{\partial}{\partial x}, 1 \leq i \leq m$, D. Heath [11], [13] shows that $\left(u_{i}(t)\right)$ has representation

$$
\begin{aligned}
u_{i}(t)= & E_{i}\left[\Gamma\left(X_{0}, T_{1}\right) \ldots \Gamma\left(X_{N(t)}, t-T_{N(t)}\right) f_{Y(t)} .\right. \\
& \left.\exp \left(\int_{0}^{t} d\left(Y_{s}\right) d s\right) \Pi_{1 \leq i \not j \leq m}\left(\rho_{i j}\right)^{N_{i j}(t)} f_{Y(t)}\right]
\end{aligned}
$$

where $\operatorname{MRP}(X, T)$ has parameters $p_{i i}=0$,

$$
p_{i j}=\left|a_{i j}\right| /\left(\sum_{k ; k \neq i}\left|a_{i k}\right|\right), j \neq i, \eta_{i}=\sum_{k ; k \neq i}\left|a_{i k}\right|, \quad 1 \leq i, j \leq m,
$$

and hence the associated SMP $Y=\left\{Y_{t} ; t \in R_{+}\right\}$is a Markov process with respect to $\sigma$-algebras $\sigma\left(Y_{s} ; 0 \leq s \leq r\right), \quad r \in R_{+}$; here $d(i)=a_{i i}+$ $\sum_{k ; k+i}\left|a_{i k}\right|, 1 \leq i \leq m$, and $\rho_{i_{j}}=\operatorname{sign}\left(a_{i j}\right), 1 \leq i \neq j \leq m$. In the setting of (3. 7), with general $A_{i}, 1 \leq i \leq m, \mathrm{M}$. Pinsky [27] shows that $\left(u_{i}(t)\right)$ has representation (3.10) where the MRP has parameters 


$$
p_{i i}=0,0 \leq p_{i j} \leq 1, \sum_{j=1}^{m} p_{i j}=1, \eta_{i} \geq 0,
$$

chosen along with the $\left\{\rho_{i j}\right\}_{1 \leq i+j \leq m}$ so that $\eta_{i} p_{i j} \rho_{i j}=a_{i j}, \quad 1 \leq i \neq j \leq m$, and $d(i)=a_{i i}+\eta_{i}$. In Pinsky's representation there is a wider possible choice of parameters for the MRP, although again the associated SMP $Y$ is Markovian with respect to $\sigma\left(Y_{s} ; 0 \leq s \leq r\right), r \in R_{+}$and essentially the same multiplicative functional is used. In the HeathPinsky representation (3.10), the form of the equations (3.7) is essentially being changed so that the coefficients in the 'coupling' part compose an infinitesimal generator of a Markov process, with the compensation for this causing additional growth in the 'streaming' part. In the representation (3.8) there are more possibilities for the effect of the underlying MRP on the total evolution, because here a multiplicative factor can also be introduced at regenerative epochs at which 'passage' from a state to itself occurs, and there is no carry-over to the 'streaming' part.

The following application demonstrates the usefulness of the concept of a R. E. with underlying MRP in generating and analyzing limitt heorems for Cauchy problems.

Application 3. 1. We first define the R. E. $M$ with underlying $\operatorname{MRP}(X, T)$ of interest, on the Banach space $L=C_{B}(R)=$ uniformly continuous, bounded functions on $R$. We let $A$ be the generator of the strongly continuous, contraction semigroup $\Gamma(u)$, defined on $L$ by

$$
\Gamma(u) f(x)=\int_{-\infty}^{\infty} f(y) k_{u}(x-y) d y
$$

where

$$
k_{u}(z)=\frac{1}{\sqrt{2 \pi u}} \exp \left(-z^{2} / 2 u\right) .
$$

For $i=1,2$ we let $\Gamma_{i}(u)=\Gamma\left(\alpha_{i} u\right)$, having generator $A_{t}=\alpha_{i} A$, where $\alpha_{i}, i=1,2$, are positive parameters with each $\alpha_{i} \rightarrow+\infty$, and $\alpha_{1}=o\left(\alpha_{2}\right)$. We let $\Pi_{i j}, 1 \leq i, j \leq 2$, denote multiplication operators defined on $L$ by $\Pi_{i j} f(x)=\pi_{i j} f(x)$, where $\left(\pi_{i j}\right)$ is given by 


$$
\left(\pi_{i j}\right)=\left(\begin{array}{rr}
4 & -2 \\
1 & 1
\end{array}\right)
$$

We use the MRP's $\left(X^{\eta}, T^{\eta}\right)$ with state space $E=\{1,2\}$, defined as those MRP's with parameters $\eta_{i}=\alpha_{1} \alpha_{i} / a, i=1,2, a>0$, and $\left(p_{i j}\right)$ given by

$$
\left(p_{i j}\right)=\left(\begin{array}{cc}
\frac{1}{2} & \frac{1}{2} \\
1 & 0
\end{array}\right) .
$$

Through definition (2.1) and the form of the operators and process defined through (3.11)-(3.14) we obtain the random evolution $M$ on $L$ given by

$$
M(t) f=\Gamma\left(\alpha_{1} \gamma_{1}^{\eta}(t)+\alpha_{2} \gamma_{2}^{\eta}(t)\right) f \cdot(4)^{N_{11}^{\eta}(t)}(-2)^{N_{12}^{\eta}(t)}
$$

where $\gamma_{i}^{\eta}(t)$ and $N_{i j}^{\eta}(t)$ are 'occupation-time' and 'number-of-jumps' random variables defined in Section one.

We give a limit theorem for R. E. $M$ defined in (3.15).

Theorem 3. 3. Let $\left(w_{j}(x, t)\right)_{j=1,2}$ denote the solution of

$$
\begin{aligned}
& \frac{\partial w_{1}}{\partial t}=\frac{\alpha_{1}}{2} \frac{\partial^{2} w_{1}}{\partial x^{2}}+\eta_{1} w_{1}-\eta_{1} w_{2} \\
& \frac{\partial w_{2}}{\partial t}=\frac{\alpha_{2}}{2} \frac{\partial^{2} w_{2}}{\partial x^{2}}+\eta_{2} w_{1}-\eta_{2} w_{2} \\
& w_{j}(0)=f .
\end{aligned}
$$

Then $w_{j}$ has representation $w_{j}(x, t)=E_{j}[M(t) f(x)]$ where $M(t)$ is the R. E. defined in (3.15). Moreover, we have

$$
\lim _{\substack{\alpha_{1}, \alpha_{2} \rightarrow \infty \\ \alpha_{1}=0\left(\alpha_{2}\right)}}\left\|w_{j}(t)-u(t)\right\|=0
$$

for $u(x, t)$ satisfying

$$
\begin{aligned}
& \frac{\partial u}{\partial t}=-a \frac{\partial^{4} u}{\partial x^{4}} \\
& u(x, 0)=f(x) .
\end{aligned}
$$

with $f \in D\left(A^{2}\right)$. 
Proof. From Theorem 3.2 we have that the solution $\left(w_{j}(x, t)\right)_{j=1,2}$ has representation $w_{j}(x, t)=E_{j}[M(t) f(x)]$ for $M(t)$ given in (3.15). The limiting result (3.17) is given as an application of abstract semi-group theorems in [20] for $f \in \mathscr{D}\left(A^{2}\right)$, and is proved by transformanalysis techniques for a smaller class of functions $f$ in [24].

Using techniques from transform analysis, we show in Theorem 3. 4 that a random evolution related to R. E. $M$ has expectation semigroup, satisfying (3.34), which also converges to the solution of (3. 18).

We let $\tau^{\eta}=\inf \left\{u \geq T_{1} ; Y^{\eta}(u)=1\right\}$. Then $\tau^{\eta}$ is an $\mathscr{H}_{s}$-stopping time. We define the operator $S_{\alpha}: L \rightarrow L$ by

$$
S_{a} f=E_{1}\left[M\left(\tau^{\eta}\right) f\right] \text {. }
$$

We prove representation and limit theorems for $S_{\alpha}$. First, we state the following lemma.

Lemma 3.1. Let $\gamma: R \rightarrow R$ denote the function

$$
r(w)=\frac{1}{4}(3-|w|) e^{-|w|} .
$$

Then the Fourier transform of $\gamma$ is given by

$$
\phi(u)=\left(1+2 u^{2}\right) /\left(1+u^{2}\right)^{2} .
$$

In particular, $\phi$ satisfies

$$
\begin{array}{ll}
\text { (i) } & 0<\phi(u) \leq 1, \phi(0)=1 \\
\text { (ii) } & \phi^{(j)}(0)=0, j=1,2,3, \quad \phi^{(4)}(0)=-4 !
\end{array}
$$

Lemma 3. 2. Given R. E. $M$ with $\operatorname{MRP}\left(X^{\eta}, T^{\eta}\right), \tau^{\eta}$, and $S_{a}$ as above. Let $\left\{R_{\mu} ; \mu \geq 0\right\}$ denote the resolvent of $\{\Gamma(u) ; u \geq 0\}$ and $A_{\mu}=A \mu R_{\mu}, \mu \geq 0$. Then $S_{\alpha}$ has the following representations:

$$
\begin{aligned}
& S_{\alpha} f=2 \lambda R_{\lambda} f-\left(\lambda R_{\lambda}\right)^{2} f \\
& S_{\alpha} f=\left(I-\lambda^{2} A_{\lambda}^{2}\right) f \\
& S_{\alpha} f(x)=\int_{-\infty}^{\infty} f(x+(w / \sqrt{2 \lambda})) \gamma(w) d w
\end{aligned}
$$


where $\lambda=\alpha_{1} / a$ and $\gamma(w)$ is given in (3.20).

Proof. For (3.23) we observe that, for $\lambda=\alpha_{1} / a$,

$$
\begin{aligned}
& E_{1}\left[M\left(\tau^{\eta}\right) f\right]=E_{1}\left[M\left(\tau^{\eta}\right) f ; \tau^{\eta}=T_{1}\right]+E_{1}\left[M\left(\tau^{\eta}\right) f ; \tau^{\eta}=T_{2}\right] \\
& =\int_{0}^{\infty} \frac{1}{2} \eta_{1} e^{-\eta_{1} s}(4) \Gamma\left(\alpha_{1} s\right) f d s+\int_{0}^{\infty} \int_{0}^{\infty} \frac{1}{2} \eta_{1} e^{-\eta_{1} s} \eta_{2} e^{-\eta_{2} u}(-2) \\
& \Gamma\left(\alpha_{1} s\right) \Gamma\left(\alpha_{2} u\right) f d s d u
\end{aligned}
$$

For (3.24) use the defining property of resolvents, i. e., $(\lambda I-A) R_{\lambda} f=f$. To prove (3.25), we need that

$$
\int_{0}^{\infty} e^{-\lambda t} k_{t}(z) d t=\sqrt{2 \lambda} \exp (-|z| \sqrt{2 \lambda}) \text { and that } \frac{d}{d \lambda} R_{\lambda}=-R_{\lambda}^{2}
$$

(see Pazy [26]). We obtain

$$
\begin{aligned}
& 2 \lambda R_{\lambda} f(x)-\left(\lambda R_{\lambda}\right)^{2} f(x)=2 \lambda R_{\lambda} f(x)+\lambda^{2} \frac{d}{d \lambda} R_{\lambda} f(x) \\
& =2 \lambda \int_{0}^{\infty} e^{-\lambda t} T(t) f(x) d t+\lambda^{2} \frac{d}{d \lambda} \int_{0}^{\infty} e^{-\lambda t} T(t) f(x) d t \\
& =\int_{-\infty}^{\infty} f(y)\{\sqrt{2 \lambda} \exp (-\sqrt{2 \lambda}|y-x|) \\
& \left.\quad+\lambda^{2} \frac{d}{d \lambda}[\sqrt{2 \lambda} \exp (-\sqrt{2 \lambda}|y-x|)]\right\} d y \\
& =\int_{-\infty}^{\infty} f(y)\left\{\frac{3}{4} \sqrt{2 \lambda}-\frac{1}{2} \lambda|y-x|\right\} \exp (-\sqrt{2 \lambda}|y-x|) d y \\
& =\int_{-\infty}^{\infty} f(x+(w / \sqrt{2 \lambda})) \gamma(w) d w .
\end{aligned}
$$

For the concepts from transform analysis used in the following lemmas see Zemanian, Chapter 7 [31]. In particular we let

$$
\begin{aligned}
\mathscr{S}= & \{f: R \rightarrow C ; f \text { is infinitely differentiable and, as }|t| \rightarrow \infty, f \\
& \text { and all its derivatives decrease to zero faster than every } \\
& \text { power of } 1 /|t|\} \\
\mathscr{S}_{R}= & \{f \in \mathscr{S} ; f \text { is real-valued }\} \\
\mathscr{S}^{\prime}= & \{\text { continuous, linear functionals on } \mathscr{S}\}
\end{aligned}
$$

$\mathscr{S}$ is the class of functions of rapid descent and $\mathscr{S}^{\prime}$ is the collection of distributions of slow growth, the tempered distributions. Note 
that $\mathscr{S}_{R}$ contains all real-valued infinitely-differentiable functions of compact support on $R$.

Lemma 3. 3. Suppose that $\rho(u)$ is defined as

$$
\rho(u)=\frac{1}{2 \pi} \int_{-\infty}^{\infty} e^{-i u x} e^{-x^{4}} d x,-\infty<u<\infty .
$$

Let $\phi \in \mathscr{S}^{\prime}$ satisfy properties (3.21), (i) and (ii), with

$$
\beta(u)=\frac{1}{2 \pi} \int_{-\infty}^{\infty} e^{-i u x} \phi(x) d x .
$$

Then

$$
\begin{aligned}
& \lim _{m \rightarrow \infty} \sup _{x} \mid \int_{-\infty}^{\infty} h\left(x+\frac{w}{m^{1 / 4}}\right) \beta^{* m}(w) d w \\
& \quad-\int_{-\infty}^{\infty} h(x+u) \rho(u) d u \mid=0
\end{aligned}
$$

for each $h \in \mathscr{S}_{R}$, where $\beta^{* m}$ denotes the mth convolution of $\beta$.

Proof. We use transform analysis for tempered distributions and test functions of rapid descent. In particular, we use Parseval's equation and a Taylor expansion of $\phi(u)$ to obtain for $h \in \mathscr{S}_{R}$ that

$$
\begin{aligned}
& \left|\int_{-\infty}^{\infty} h\left(x+\frac{w}{m^{1 / 4}}\right) \beta^{* m}(w) d w-\int_{-\infty}^{\infty} h(x+w) \rho(w) d w\right| \\
& \quad=\left|\int_{-\infty}^{\infty} h(u)\left\{m^{1 / 4} \beta^{* m}\left(m^{1 / 4}(u-x)\right)-\rho(u-x)\right\} d u\right| \\
& \quad=\left|\int_{-\infty}^{\infty} \hat{h}(u)\left\{\left(\phi\left(\frac{u}{m^{1 / 4}}\right)\right)^{m}-e^{-u^{4}}\right\} e^{-i u x} d u\right| \\
& \quad \leq \int_{-\infty}^{\infty}|\hat{h}(u)|\left|\left(\phi\left(\frac{u}{m^{1 / 4}}\right)\right)^{m}-e^{-u^{4}}\right| d u .
\end{aligned}
$$

Since $h \in \mathscr{S}_{R}$ implies $\hat{h} \in \mathscr{S}$, we know that $\int_{-\infty}^{\infty}|\hat{h}(u)| d u<\infty$; thus the integrand is majorized by the integrable function $2|\hat{h}|$. From the assumptions on $\phi$ we obtain for each $u \in R^{1}$

$$
\begin{aligned}
& \left.\lim _{m \rightarrow \infty}\left(\phi\left(\frac{u}{m^{1 / 4}}\right)\right)\right)^{m}-e^{-u^{4}} \\
& \quad=\lim _{m \rightarrow \infty} \exp \left\{m \cdot \ln \phi\left(\frac{u}{m^{1 / 4}}\right)\right\}-e^{-u^{4}}
\end{aligned}
$$




$$
\begin{aligned}
& =\lim _{m \rightarrow \infty} \exp \left\{m\left(\phi\left(\frac{u}{m^{1 / 4}}\right)-1\right)\left(1+O\left(\phi\left(\frac{u}{m^{1 / 4}}\right)-1\right)\right)\right\}-e^{-u^{4}} \\
& =\lim _{m \rightarrow \infty} \exp \left\{m\left(-\frac{u^{4}}{m}+O\left(u, \frac{1}{m^{5 / 4}}\right)\right)\left(1+O\left(\frac{1}{m}\right)\right)\right\}-e^{-u^{4}} \\
& =0
\end{aligned}
$$

We thus can apply the Lebesgue dominated convergence theorem to obtain (3.26) from (3.27).

In [15], Hochberg mentions that he has obtained the result $\lim _{m \rightarrow \infty} \int_{-\infty}^{\infty} h\left(\frac{w}{m^{1 / 4}}\right) \beta^{* m}(w) d w=\int_{-\infty}^{\infty} h(u) \rho(u) d u$ for functions $h$ of rapid descent with $\hat{h}$ of compact support. Since his proof is not easily accessible at this time, we included our own proof in some detail. Miyamoto [24] and Krylov [22] also give theorems closely related to Lemma 3.3.

Lemma 3.4. For each $f \in \mathscr{S}_{R}$, we have

$$
\lim _{\lambda \rightarrow \infty}\left\|S_{\lambda}^{\lambda^{2} a t} f-T(t) f\right\|=0
$$

where $\lambda=\alpha_{1} / a \rightarrow \infty$ with $\lambda^{2}$ at taking integer values. Here $u(x, t)=$ $T(t) f(x)$ satisfies the initial value problem (3.18).

Proof. We have the representations

$$
\begin{aligned}
S_{\lambda}^{\lambda^{2} a t} f(x) & =\int_{-\infty}^{\infty} f\left(x+\frac{w}{\sqrt{2 \lambda}}\right) \gamma^{* \lambda^{2} a t}(w) d w \\
T(t) f(x) & =\int_{-\infty}^{\infty} f(x+s) g_{t}(s) d s \\
& =\int_{-\infty}^{\infty} f\left(x+\frac{w}{\sqrt{2 \lambda}}\right) g_{1}^{* 2^{2} t}(w) d w .
\end{aligned}
$$

For (3.29) we use function $\gamma$ given in (3.20); the verification of (3.29) uses (3.25) and an induction argument. For (3.30) we use

$$
g_{i}(u)=\frac{1}{2 \pi} \int_{-\infty}^{\infty} e^{-i u x} \psi_{t}(x) d x
$$

where $\phi_{t}(x)=\exp \left(-\mathrm{a} x^{4} t\right)$. The representation (3.25) is given in 
Hersh [12] and Donaldson-Hersh [8]; transform techniques give the second form. (3.28) now follows directly from Lemmas (3.1) and (3.3).

Lemma 3. 5. For each $f \in \mathscr{S}_{R}$, we have

$$
\lim _{\lambda \rightarrow \infty}\left\|E_{1}\left[S_{\lambda}^{N_{2}(t)} f\right]-T(t) f\right\|=0
$$

where $\lambda=\alpha_{1} / a \rightarrow \infty$ with $\lambda^{2}$ at taking integer values, $N_{\lambda}(t)=$ number of times $Y^{n}$ enters state one before time $t=N_{1,1}^{2}(t)+N_{2,1}^{2}(t)$, and $T(t) f$ is given in Lemma 3. 4 .

Proof. From (3.29) and Parseval's equation we have for $f \in \mathscr{S}_{R}$ that

$$
\begin{aligned}
& \left|E_{1}\left[S_{\lambda}^{N_{2}(t)} f(x)\right]-S_{\lambda}^{2^{2} a t} f(x)\right| \\
& =\mid E_{1}\left[\int_{-\infty}^{\infty} f\left(x+\frac{w}{\sqrt{2 \lambda}}\right) \gamma^{* N_{\lambda}(t)}(w) d w\right] \\
& \quad-\int_{-\infty}^{\infty} f\left(x+\frac{w}{\sqrt{2 \lambda}}\right) r^{* \lambda^{2} a t}(w) d w \mid \\
& =\left|E_{1}\left[\int_{-\infty}^{\infty} \hat{f}(u)\left\{\left(\phi\left(\frac{u}{\sqrt{2 \lambda}}\right)\right)^{N_{2}(t)}-\left(\phi\left(\frac{u}{\sqrt{2 \lambda}}\right)\right)^{2^{2} a t}\right\} e^{-i u x} d u\right]\right| \\
& \leq \int_{-\infty}^{\infty}|\hat{f}(u)| E_{1}\left\{\left|\left(\phi\left(\frac{u}{\sqrt{2 \lambda}}\right)\right)^{N_{2}(t)}-\left(\phi\left(\frac{u}{\sqrt{2 \lambda}}\right)\right)^{2^{2} a t}\right|\right\} d u .
\end{aligned}
$$

As in Lemma 3.3, the last integrand is majorized by $2|\hat{f}(u)|$. We also have that the integrand goes to zero pointwise as $\lambda \rightarrow \infty$, since

$$
\begin{aligned}
& \lim _{\lambda \rightarrow \infty} E\left\{\left|\left(\phi\left(\frac{u}{\sqrt{2 \lambda}}\right)\right)^{N_{\lambda}(t)}-\left(\phi\left(\frac{u}{\sqrt{2 \lambda}}\right)\right)^{2^{2} \alpha t}\right|\right\} \\
& \left.\leq \lim _{\lambda \rightarrow \infty} E\left\{\mid\left(\phi\left(\frac{u}{\sqrt{2 \lambda}}\right)\right)\right)^{\left|N_{\lambda}(t)-\lambda^{2} a t\right|}-1 \mid\right\} \\
& =\lim _{\lambda \rightarrow \infty} E\left\{\mid\left(1+\phi\left(\frac{u}{\sqrt{2 \lambda}}\right)+\ldots+\phi\left(\frac{u}{\sqrt{2 \lambda}}\right){ }^{\left|N_{2}(t)-\lambda^{2} a t\right|-1}\right)\right. \\
& \left.\left.\qquad \phi\left(\frac{u}{\sqrt{2 \lambda}}\right)-1\right) \mid\right\} \\
& \leq \lim _{\lambda \rightarrow \infty} E\left\{\left|N_{2}(t)-\lambda^{2} a t\right|\right\}\left|\phi\left(\frac{u}{\sqrt{2 \lambda}}\right)-1\right|
\end{aligned}
$$




$$
\begin{aligned}
& \left.\leq \lim _{\lambda \rightarrow \infty} E\left\{\left(N_{\lambda}(t)-\lambda^{2} a t\right)\right)^{2}\right\}^{1 / 2}\left|\phi\left(\frac{u}{\sqrt{2 \lambda}}\right)-1\right| \\
& =\lim _{\lambda \rightarrow \infty} c\left(\lambda^{2} a t\right)^{1 / 2}\left|\frac{w^{4}}{\lambda^{2}} \phi^{(4)}\left(\frac{\theta w}{\sqrt{2 \lambda}}\right)\right| \\
& =0 .
\end{aligned}
$$

Here we have used that $E\left[\left(N_{\lambda}(t)-\lambda^{2} a t\right)^{2}\right] \sim \lambda^{2} a t$ as $\lambda \rightarrow \infty$ (see [7]). Thus by the Lebesgue dominated convergence theorem we have from (3. 32)

$$
\lim _{\lambda \rightarrow \infty}\left\|E_{1}\left[S_{\lambda}^{N_{2}(t)} f\right]-S_{\lambda}^{\lambda^{2} a t} f\right\|=0
$$

From (3.33) and Lemma 3.5, we have (3.31) follows.

Theorem 3. 4. Suppose $\left(v_{j}^{\lambda}(x, t)\right)_{j=1,2}$ denotes the solution of

$$
\begin{aligned}
& \frac{\partial v_{1}}{\partial t}=-\frac{1}{2} \eta_{1} v_{1}+\frac{1}{2} \eta_{1} v_{2}+\frac{1}{2} \eta_{1}\left(S_{2}-I\right) v_{1} \\
& \frac{\partial v_{2}}{\partial t}=\eta_{2} v_{1}-\eta_{2} v_{2}+\eta_{2}\left(S_{2}-I\right) v_{1} \\
& v_{j}(0)=f
\end{aligned}
$$

where $\eta_{1}=\alpha_{1}^{2} / a, \eta_{2}=\alpha_{1} \alpha_{2} / a$, and $\lambda=\alpha_{1} / a$ and $u(x, t)$ denotes the solution of (3.18).

Then for $f \in \mathscr{S}_{R}$ we have that

$$
\lim _{\alpha_{1}, \alpha_{2} \rightarrow \infty}\left\|v_{j}(t)-u(t)\right\|=0
$$

for $j=1,2$.

Proof. We observe that (3.34) can be written in the form of (3.6) by taking $\left(p_{i j}\right)$ as in (3.14), $A_{i}=0$ for $i=1,2, \Pi_{11}=\Pi_{21}=S_{2}$ and $\Pi_{12}=\Pi_{22}=I$. Hence from (2.1) and part (3) of Theorem 3.2 we have that the solution $\left(v_{j}(x, t)\right)_{j=1,2}$ for (3.31) has representation $v_{j}(x, t)=E_{j}\left[S^{N_{\lambda}(t)} f(x)\right]$ for $j=1,2$. From Lemma 3.5 we have that $\lim _{\alpha_{1}, \alpha_{2} \rightarrow \infty}\left\|v_{1}(t)-u(t)\right\|=0$. Finally we have that

$$
\begin{aligned}
v_{2}(x, t) & =E_{2}\left[S_{2}^{N_{\lambda}(t)} f(x)\right] \\
& =e^{-\eta_{2} t} f(x)+\int_{0}^{t} \eta_{2} e^{-\eta_{2} r} S_{\lambda} v_{1}(x, t-r) d r .
\end{aligned}
$$


Thus

$$
\begin{aligned}
v_{2}(x, t)-v_{1}(x, t)=e^{-\eta_{2} t} f(x)+\int_{0}^{\eta_{2} t} e^{-r} & \left\{S_{\lambda} v_{1}\left(x, t-\frac{r}{\eta_{2}}\right)\right. \\
& \left.-v_{1}(x, t)\right\} d r-e^{-\eta_{2} t} v_{1}(x, t) .
\end{aligned}
$$

By using transform analysis on the integral on the right-hand side as in Lemma 3.3 and 3.5, we obtain

$$
\lim _{\alpha_{1}, \alpha \rightarrow \infty}\left\|v_{2}^{\lambda}(t)-v_{1}^{\lambda}(t)\right\|=0 .
$$

From Lemma 3.5 we conclude that $\lim _{\alpha_{1}, \alpha_{2} \rightarrow \infty}\left\|v_{2}^{2}(t)-u(t)\right\|=0$.

\section{Bibliography}

[1] Bharucha-Reid, A.T., Random Integral Equations, Academic Press, New York (1972).

[2] Blumenthal, R.M. and Getoor, R. K., Markov Processes and Potential Theory, Academic Press, New York (1968).

[3] Breiman, L., Probability, Addison-Wesley, London (1968).

[4] Çinlar, E., On semi-Markov processes on arbitrary spaces, Proc. Camb. Phil. Soc., 63 (1969) 381-392.

[5] On dams with continuous semi-Markovian inputs. J. Math. Anal. Appl., 35 (1971) 434-448.

[6] Introduction to Stochastic Processes, Prentice-Hall, Inc., Englewood Cliffs, N. J. (1975).

[7] Cox, D. R., Renewal Theory, Wiley, New York (1962).

[8] Donaldson, J. A. and Hersh, R., A perturbation series for Cauchy's problem for higher-order abstract parabolic equations, Proc. Nat. Acad. Sci. U.S. A., 67 (1970) 4144.

[9] Griego, R. and Hersh, R., Theory of random evolutions with applications to partial differential equations, Trans. A mer. Math. Soc., 156 (1971) 405-418.

[10] Griego, R. and Moncayo, A., Random evolutions and piecing out of Markov processes Bol. Soc. Mat. Mexicana, 15(1970) 22-29.

[11] Heath, D., Probabilistic analysis of certain hyperbolic systems of partial differential equations, Ph. D. dissertation, Univ. of Illinois (1969).

[12] Hersh, R., Direct solution of general one-dimensional linear parabolic equation via an abstract Plancherel formula, Proc. Nat. Acad. Sci. U.S. A., 63 (1969) 648-654.

[13] , Random evolutions : a survey of results and problems, Rocky Mountain Math. J., 4 (1974) 443-477.

[14] Hille, E. and Phillips, R. S., Functional Analysis and Semigroups, rev. ed., Amer. Math. Soc. Colloq. Publ., 31, Amer. Math. Soc., Providence, R. I. (1957).

[15] Hochberg K., Summary of Ph. D. dissertation, New York University (1976).

[16] Ito, K. and McKean, Jr., H. P., Diffusion Processes and Their Sample Paths, SpringerVerlag, New York (1965).

[17] Kato, T., Perturbation Theory for Linear Operators, Springer-Verlag, New York (1966).

[18] Kertz, R. P., Limit theorems for discontinuous random evolutions with applications to initial value problems and to Markov processes on $N$ lines. Ann. Probability, 2 (1974) 
1046-1064.

[19] — Perturbed semigroup limit theorems with applications to discontinuous random evolutions Trans. A mer. Math. Soc., 199 (1974) 29-53.

[20] L Limit theorems for semigroups with perturbed generators, with applications to multi-scaled random evolutions, Functional Analysis, 27 (1978) 215-233

[21] , Random evolutions in a semi-Markov model of Brownian motion (unpublished manuscript).

[22] Krylov, V. Yu., Some properties of the distribution corresponding to the equation $\frac{\partial u}{\partial t}=(-1)^{q+1} \frac{\partial^{2 \ell} u}{\partial x^{2 \ell}}$, Doklady, 132 (1960) 1254-1257.

[23] Kurtz, T. G., Comparison of semi-Markov and Markov processes, Ann. Math. Statist., 42 (1971) 991-1002.

[24] Miyamoto, M., An extension of certain quasi-measure, Proc. Japan Academy, 42 (1966) 70-74.

[25] Mürman, M.G., A semi-Markovian model for the Brownian motion. Seminaire de Probabilitiés VII, Lecture Notes in Math., 321, 248-272, Springer-Verlag.

[26] Pazy, A., Semigroups of linear operators and applications to partial differential equations, Lecture Notes 10, Univ. of Maryland (1974).

[27] Pinsky, M., Multiplicative Operator Functionals and Their Asymptotic Properties, Advances in probability III, Marcel Dekker, New York (1974).

[28] Scalora, F.S. Abstract martingale convergence theorems, Pacific J. Math., 2 (1961) 347-374.

[29] Schäl, M. Markov renewal processes with auxiliary paths, Ann. Math. Statist., 41(1970) 1604-1623.

[30] Smith, W.L., Regenerative stochastic processes. Proc. Roy. Soc. Ser. A., 232 (1955) 6-31.

[31] Zemanian, A.H., Distribution Theory and Transform Analysis, McGraw-Hill, New York (1965). 